\title{
Association between TAp73, p53 and VASH1 expression in lung adenocarcinoma
}

\author{
MENG WU ${ }^{1,2}$, ZHIHUA ZHANG $^{2}$, FANGXU MA $^{2}$, XIULONG ZHANG $^{2}$, ZHILIN ZHANG $^{2}$, \\ JIANHUA TANG $^{2}$, PING CHEN ${ }^{1}$, CHUNYAN ZHOU $^{1}$ and WEIPING WANG ${ }^{1}$
}

\author{
${ }^{1}$ Department of Biochemistry and Molecular Biology, School of Basic Medical Sciences, \\ Key Laboratory of Molecular Cardiovascular Sciences, Ministry of Education of China, Peking University, Beijing 100191; \\ ${ }^{2}$ Department of Respiration, First Affiliated Hospital of Hebei North University, Zhangjiakou, Heibei 075061, P.R. China
}

Received December 27, 2016; Accepted January 9, 2018

DOI: $10.3892 / 01.2018 .7912$

\begin{abstract}
TAp73 and p53 are involved in regulating tumor angiogenesis and vasohibin-1 (VASH1) is an anti-angiogenic factor. Whether TAp73 regulates angiogenesis positively or negatively is controversial. The status of P53 may determine the effect of TAp73 on angiogenesis. To the best of our knowledge it has not been previously reported whether TAp73, p53 and VASH1 are coexpressed in lung cancer. We profiled the association between TAp73 and p53 and VASH1 expression in lung adenocarcinoma (LAC) and investigated the function of TAp73 in regulating tumor angiogenesis. TAp73, p53 and VASH1 expression in 53 human LAC tissues and the adjacent normal tissues were evaluated using immunohistochemistry. The positive expression rates of p53, TAp73 and VASH1 were significantly higher (92.6, 97.7 and $67.4 \%$, respectively) in LAC tissue compared with paraneoplastic lung tissue (7.4, 2.3 and $32.6 \%$, respectively, $\mathrm{P}<0.01$ ). Pearson's correlation coefficient showed a significant positive correlation between $\mathrm{p} 53$ and TAp73 $(r=0.474, \mathrm{P}<0.01)$ and TAp73 $\alpha$ and VASH1 $(r=0.367$, $\mathrm{P}<0.01)$. The positive expression rate of $\mathrm{p} 53$ and VASH1 was almost significantly correlated $(\mathrm{r}=0.187, \mathrm{P}=0.055)$. Similarly, p53 expression intensity had a significant positive correlation with TAp73 $\alpha(\mathrm{r}=0.517, \mathrm{P}<0.01)$ and with VASH1 $(\mathrm{r}=0.277$, $\mathrm{P}<0.01)$, as did TAp73 $\alpha$ with VASH1 $(\mathrm{r}=0.351, \mathrm{P}<0.01)$. TAp73, p53 (mutant) and VASH1 expression was significantly higher in LAC tissue compared with paraneoplastic lung tissue. The
\end{abstract}

Correspondence to: Dr Zhihua Zhang, Department of Respiration, First Affiliated Hospital of Hebei North University, 12 Changqing Road, Zhangjiakou, Heibei 075061, P.R. China

E-mail: zzh19641229@163.com

Dr Weiping Wang, Department of Biochemistry and Molecular Biology, School of Basic Medical Sciences, Key Laboratory of Molecular Cardiovascular Sciences, Ministry of Education of China, Peking University, 38 Xueyuan Road, Beijing 100191, P.R. China E-mail:wwp@bjmu.edu.cn

Key words: TAp73, p53, vasohibin-1, lung adenocarcinoma, angiogenesis, immunohistochemistry expression trends of the three proteins were significantly positively correlated with each other in LAC. These results suggest that TAp73 may suppress tumor angiogenesis in LAC.

\section{Introduction}

Lung cancer is one of the five leading causes of cancer death worldwide. The incidence of pulmonary cancer has been increasing steadily in both men and women in the developing countries, including China, by the year. Non-small cell lung cancer (NSCLC), which is aggressive and has a poor prognosis, accounts for approximately $75-85 \%$ of lung cancers (1). The proportion of lung adenocarcinoma (LAC), a common form of NSCLC, is also increasing. Therefore, in-depth research of the pathogenesis of LAC would be of significance.

Tumor angiogenesis is one of the most important hallmarks in the development of lung cancer. An extensive microvascular system is closely related to the malignant behaviors of LAC, and is also an important target in LAC treatment (2). p73, a homologue of the tumor suppressor p53, regulates vascular development and cell proliferation, migration, and differentiation. There are two isoforms of p73: TAp73, which contains full-length $\mathrm{N}$-terminal transactivating domains; it is also termed TAp73 $\alpha$. The other is the $\Delta \mathrm{Np} 73$ isoform, which has a truncated $\mathrm{N}$-terminal and promotes cancer gene programs (3). P73-knockout (TAp73-/-) mice develop gastrointestinal and cranial hemorrhage (4), indicating vascular fragility. In addition, TAp73 regulates GATA-1 (5), which is critical for endothelial and hematopoietic cell differentiation. Accordingly, we conjectured that p73 might be related to vasculogenesis/angiogenesis. However, the function of TAp73 in regulating angiogenesis in cancer is hotly debated $(6,7)$. Some suggest that TAp73 suppresses angiogenesis $(8,9)$. Conversely, others suggest that both the TAp73 and $\Delta \mathrm{Np} 73$ forms are proangiogenic (10-12). Interestingly, p53 status might be a determinant of the effect of TAp73 on angiogenesis. TAp73 regulated vascular endothelial growth factor A (VEGFA), where wild-type p53 downregulated angiogenesis and mutant p53 promoted it (13). Altogether, whether TAp73 acts as a positive or negative regulator of angiogenesis is unclear.

The tumor suppressor gene TP53 has been described as a critical determinant of the angiogenic potential of tumor cells. 
Indeed, the TP53 gene is mutated in many human tumors. Mutated TP53 genes promote the invasion, metastasis, proliferation, and angiogenesis of malignant cells (14). In addition, mutant p53 protein upregulates VEGF and promotes angiogenesis (15). In contrast to p53, analysis of TAp73 in different human tumors did not detect any significant mutation. Rather, wild-type TAp73 is overexpressed in many human tumors, suggesting that TAp73, as well as p53, is involved in regulating tumor angiogenesis. However, whether TAp73 and P53 are coexpressed in lung cancer, especially LAC, has not been reported.

Numerous endogenous regulators participate in cancer angiogenesis. Vasohibin-1 (VASH1) is a VASH family member that has been identified as a novel negative feedback regulator of angiogenesis (16). It is expressed and secreted by the termination zone of endothelial cells. Consequently, VASH1 halts angiogenesis and exerts some anti-angiogenic activity in endothelial cells (17). High VASH1 expression has also been detected in some tumor tissues (colorectal cancer, NSCLC) and correlates positively with clinicopathological features, including lymph node status and tumor-node-metastasis (TNM) stage, and is an independent prognostic factor (18). It is well-established that VASH1 acts as an anti-angiogenic factor. Its overexpression in several tumors is due to VEGF induction. Reduced VASH1 expression has been reported in renal cell carcinoma (RCC) (19). Lu et al also reported that EZH2 silenced VASH1 expression in ovarian cancer, worsening prognosis (20). Delivery recombinant adenovirus encoding VASH1 prevents tumor angiogenesis and tumor growth (21). Although TAp73, p53, and VASH1 have been studied individually in tumors, little is known about their coexpression status in lung cancer. In the present study, we profile the association between TAp73, p53, and VASH1 expression in LAC by immunohistochemical (IHC) staining and statistical analysis. The study will be of benefit in that it provides clues to the function of TAp73 in regulating angiogenesis in LAC.

\section{Materials and methods}

Patients and tissue microarray. Microarray sections of human LAC tissues and adjacent normal tissues with pathological information were purchased from Shanghai Biochip (OD-CT-RsLug04-003; Shanghai, China). Table I lists the clinicopathological characteristics of the patients. TAp73, p53, and VASH1 protein expression was analyzed in the 53 LAC tissue samples and the paired adjacent normal tissues.

Immunohistochemistry. Tissue sections (4- $\mu \mathrm{m}$ thick) were cut from each tissue microarray block and the tissue microarrays were performed according to the streptavidin-peroxidase IHC method. The tissue sections were conventionally dewaxed, and antigen retrieval was performed by submerging the slides in sodium citrate buffer ( $\mathrm{pH}$ 6.0) under high-pressure and high-temperature conditions, 3-minute boiling, followed by 30 -min cooling at room temperature. Then, the tissue sections were incubated in hydrogen peroxide $(3 \%)$ in phosphate-buffered saline containing 1\% Triton X-100 (PBS-T) for $10 \mathrm{~min}$ to block endogenous peroxidase activity. The sections were washed repeatedly in 5\% bovine serum albumin in PBS-T and incubated with mouse polyclonal anti-VASH1 antibody
(1:100; ab67423; Abcam, Hong Kong, China), rabbit polyclonal anti-P73 antibody (1:1,000; ab14430; Abcam), and monoclonal anti-p53 antibody (1:1,000; p5813; Sigma-Aldrich; Merck KGaA, Darmstadt, Germany) in $5 \%$ bovine serum albumin at $4{ }^{\circ} \mathrm{C}$ overnight. The next day, the sites of immunoreactivity were detected using an EnVision Detection kit/DAB staining kit (GK500705; Dako A/S, Glostrup, Denmark) according to the manufacturer's protocol. The slides were counterstained with hematoxylin (ZLI-9609; ZSGB-Bio, Beijing, China) for $1 \mathrm{~min}$ at room temperature, and then dehydrated and sealed using crystal mounting solution (ZLI-9516; ZSGB-Bio).

Image analysis and scoring. Two pathologists evaluated the immunostaining independently using a double-blind method. Staining intensity was evaluated according to the following scale: 0 points, unstained; 1 point, faint yellow; 2 points, brownish yellow; 3 points, brown. Staining was also assessed according to the percentage of positive cells (0-100\%). In the tissue slides, negative and positive expression was determined by the percentage of positively stained cells and the staining intensity; score $\leq 3$ was defined as negative expression; score $>3$ was defined as positive expression (Table II). By multiplying the positively stained cells by the staining intensity, the protein expression intensity was classified on a subjective spectrum as follows: -, 0-1 points;,$+ 2-4$ points;,$++ 5-8$ points; +++ , $>9$ points (Table III).

Statistical analysis. All statistical analyses were performed using SPSS (version 21.0; SPSS, Inc., Chicago, IL, USA). The clinical characteristics of the patients according to negative and positive expression of p53, TAp73, and VASH1 were assessed using the chi-square test and Student's t-test. Spearman's correlation was used to determine the relationship between positive and negative protein expression and with expression intensity. $\mathrm{P}<0.05$ was considered to indicate a statistically significant difference.

\section{Results}

Study population. Table I summarizes the demographic and clinicopathological features of the samples, which included 53 LAC tissue samples and 53 paraneoplastic tissue samples. The mean patient age was 64.0 years, and $52.8 \%$ of the patients were male. Approximately $43.4 \%$ of the patients were smokers. Most patients had stage II LAC $(75.5 \%)$; the remaining patients had stage I (1.9\%), stage I-II (1.9\%), stage II-III (13.2\%), and stage III disease $(7.5 \%)$.

P53, TAp73, and VASH1 expression in LAC. P53, TAp73, and VASH1 expression in the LAC and paraneoplastic lung tissue was analyzed by immunohistochemistry. The expression of the proteins was classified into two groups: Negative and positive expression. P53 and TAp73 were mainly localized to the pulmonary alveoli nuclei and expressed in LAC. VASH1 staining was observed mainly in the cytoplasm of LAC cells and in the alveolar epithelium. Different staining intensities were observed for the three proteins in the LAC or paraneoplastic lung tissues (Fig. 1). The positive expression rates of p53, TAp73, and VASH1 were significantly higher in LAC tissue (92.6, 97.7 and $67.4 \%$, respectively) than in 
Table I. General patient characteristics $(n=106)$.

\begin{tabular}{lc}
\hline Feature & $\%(\mathrm{n})$ \\
\hline Mean age & $64.0(9.9)$ \\
Median age (p25-p75) & $65(56-72)$ \\
Median age (years) & \\
$\leq 60$ & $35.8(38 / 106)$ \\
$>60$ & $64.2(68 / 106)$ \\
Sex & \\
Male & $52.8(56 / 106)$ \\
Female & $47.2(50 / 106)$ \\
Smoking exposure & \\
Non-smoker & $56.6(60 / 106)$ \\
Smoker & $43.4(46 / 106)$ \\
Histological type & \\
Adenocarcinoma & $50.0(53 / 106)$ \\
Paraneoplastic & $50.0(53 / 106)$ \\
Disease stage & \\
I & $1.9(1 / 53)$ \\
I-II & $1.9(1 / 53)$ \\
II & $75.5(40 / 53)$ \\
II-III & $13.2(7 / 53)$ \\
III & $7.5(4 / 53)$ \\
\hline
\end{tabular}

I, well-differentiated adenocarcinoma; II, moderately-differentiated adenocarcinoma; III, poorly-differentiated adenocarcinoma.

paraneoplastic lung tissue $(7.4,2.3$ and $32.6 \%$, respectively, $\mathrm{P}<0.01$ ) (Tables II and III). There was a clear trend for upregulated p53, TAp73, and VASH1 expression in LAC tissue as compared to paraneoplastic lung tissue. However, there was no significant difference between p53, TAp73, and VASH1 expression and age, sex, smoking exposure, or disease stage (P>0.05) (Table II).

Correlation between positive rate of $p 53$, TAp73, and VASHI expression in $L A C$. Immunochemistry analysis showed that the positive rate and intensity of p53, TAp73, and VASH1 expression were higher in LAC tissue than in paraneoplastic lung tissue. We evaluated the correlation between the three using Pearson's correlation coefficient. There was extremely significant positive correlation for the rate of positive expression between p53 and TAp73 ( $\mathrm{r}=0.474, \mathrm{P}<0.01)$ and between TAp73 and VASH1 ( $\mathrm{r}=0.367, \mathrm{P}<0.01$; Table IV). There was a marginal significant correlation for the rate of positive expression between 53 and VASH1 ( $\mathrm{r}=0.187, \mathrm{P}=0.055)$.

Correlation between p53, TAp73, and VASH1 expression intensity in $L A C$. To further estimate the correlation between p53, TAp73, and VASH1 expression in LAC, we analyzed their expression intensity in LAC and paraneoplastic lung tissues. Similar to the rate of positive expression, p53 expression intensity had extremely significant positive correlation with TAp73 $(r=0.517, \mathrm{P}<0.01)$ and with VASH1 $(r=0.277, \mathrm{P}<0.01)$. There was also extremely significant positive correlation the TAp73 and VASH1 expression intensities $(\mathrm{r}=0.351, \mathrm{P}<0.01$; Table V). Therefore, the statistical analysis suggested a positive association between p53, TAp73, and VASH1 expression abundance in LAC.

\section{Discussion}

Approximately 140 million people die from lung cancer, particularly NSCLC, each year, and lung cancer is the most common cause of cancer-related death around the world. The molecular basis of lung cancer is complex and warrants further investigation.

The members of the p53 family include p53, p73, and p63. Wild-type p53 suppresses tumor formation. The normal protein has a very short half-life and is present in only minute amounts in normal tissues and cells. However, TP53 is the most frequently mutated gene in numerous human cancers, as recent whole-exome sequencing studies have shown (22). TP53 mutations gain new activity to promote tumor progression and angiogenesis (23). In contrast to the wild type, mutant p53 protein produced by malignant cells is usually a product of a point mutation in the TP53 gene, leading to the substitution of a single amino acid that significantly prolongs the half-life of the protein. The accumulation of high levels of p53 is a potential novel marker of malignancy. As the mutation is found in more than $60 \%$ of NSCLC cases, and the mutant protein has a longer half-life than the wild-type protein in the nucleus, the nuclear p53 protein accumulation in LAC tissues we detected by IHC is likely the mutant type. Ultimately, direct sequencing of the LAC samples would confirm the TP53 mutations.

Another member of the p53 family is TAp73. TAp73 has two main isoforms (24). One is the full-length TAp73 yielded by the $\mathrm{P} 1$ promoter, and contains the $\mathrm{N}$-terminal transactivation domain. Cell-based assays have shown that it is functionally analogous to $\mathrm{p} 53$. The other isoform is $\Delta \mathrm{Np} 73$ which lacks the $\mathrm{N}$-terminal transactivation domain and acts as a repressor of p53 and TAp73 (25). Recently, it was shown that TAp73 as well as p53 are involved in regulating tumor angiogenesis. However, the role of TAp73 in vasculogenesis remains contradictory and unclear. Some reports have suggested that TAp73 has a suppressive effect on angiogenesis $(8,9)$. Conversely, other reports suggest that TAp73 is a proangiogenic factor. Some researchers have proposed the explicit possibility that TAp 73 both promotes and inhibits angiogenesis in cancer depending on the cellular context. Transient overexpression of TAp73 leads to pro-angiogenesis. In contrast, long-term induction of TAp73 leads to angiogenic suppression (26). Similarly, we also found TAp73 could inhibit the ability of tube formation in human umbilical vein endothelial cells (HUVEC) when HUVEC co-cultured with H1299 LAC cells with TAp73 stable overexpressed not for transient transfection (data not shown). Based on this point of view, we speculate that TAp73 suppresses angiogenesis in the long-term development of cancer.

In addition, a different p53 status might affect the role of TAp73 in angiogenesis (6). Therefore, profiling the coexpression status of TAp73 and p53 in tumor cells is crucial. We observed significantly higher expression of TAp73 and mutant p53 in LAC tissues compared to paraneoplastic lung tissue. Moreover, both proteins exhibited extremely significant positive correlation expression trends. In addition to hypoxia, DNA damage 


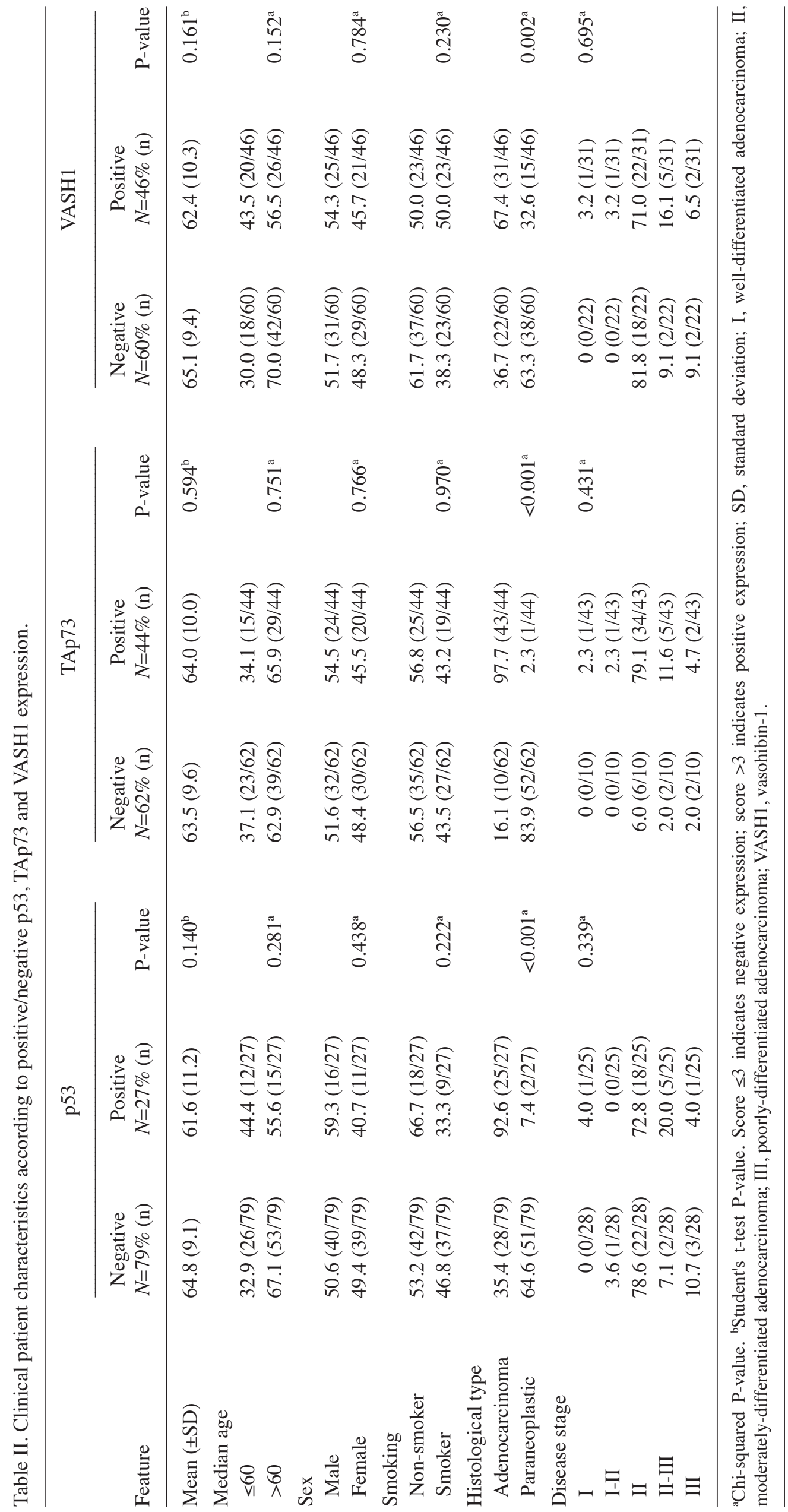


Table III. Expression intensity of p53, TAp73 and VASH1 $(n=106)$.

\begin{tabular}{|c|c|c|c|c|c|c|c|c|c|}
\hline & \multicolumn{3}{|c|}{$\mathrm{p} 53$} & \multicolumn{3}{|c|}{ TAp73 } & \multicolumn{3}{|c|}{ VASH1 } \\
\hline & LAC & Paraneoplastic & Total & LAC & Paraneoplastic & Total & LAC & Paraneoplastic & Total \\
\hline- & 21 & 49 & 70 & 7 & 43 & 50 & 9 & 19 & 28 \\
\hline+ & 12 & 4 & 16 & 22 & 10 & 32 & 26 & 30 & 56 \\
\hline++ & 14 & 0 & 14 & 21 & 0 & 21 & 13 & 4 & 17 \\
\hline+++ & 6 & 0 & 6 & 3 & 0 & 3 & 5 & 0 & 5 \\
\hline Total & 53 & 53 & 106 & 53 & 53 & 106 & 53 & 53 & 106 \\
\hline
\end{tabular}

Protein expression intensity was classified on a subjective spectrum:,- 0-1 points;,$+ 2-4$ points;,$++ 5-8$ points;,$+++>9$ points; VASH1, vasohibin-1; LAC, lung adenocarcinoma.

Table IV. Correlation between the positive expression rate of $\mathrm{p} 53$, TAp73 and VASH1 $(\mathrm{n}=106)$.

\begin{tabular}{lccc}
\hline & $\mathrm{p} 53$ & TAp73 & VASH1 \\
\hline $\mathrm{p} 53$ & & & \\
$\mathrm{r}$ & 1.000 & 0.474 & 0.187 \\
P-value & - & $<0.001$ & 0.050 \\
TAp73 & & & \\
r & 0.474 & 1.000 & 0.367 \\
P-value & $<0.001$ & - & 0.006 \\
VASH1 & & & \\
r & 0.187 & 0.367 & 1.000 \\
P-value & 0.050 & 0.006 & - \\
\hline
\end{tabular}

r, Spearman correlation; VASH1, vasohibin-1.

Table V. Correlation between p53, TAp73 $\alpha$, and VASH1 expression intensity $(\mathrm{n}=106)$.

\begin{tabular}{lccr}
\hline & $\mathrm{p} 53$ & TAp73 $\alpha$ & VASH1 \\
\hline p53 & & & \\
r & 1.000 & 0.517 & 0.277 \\
P-value & - & 0.004 & $<0.001$ \\
TAp73 $\alpha$ & & & \\
r & 0.517 & 1.000 & 0.351 \\
P-value & 0.004 & - & $<0.001$ \\
VASH1 & & & \\
r & 0.277 & 0.351 & 1.000 \\
P-value & $<0.001$ & $<0.001$ & - \\
\hline
\end{tabular}

r, Spearman correlation; VASH1, vasohibin-1.

and factors such as p53 status can be p73-mediated angiogenesis response modulators. The mechanism may be related to TAp73 regulation of VEGF in the presence of wild-type 553 conditions, where TAp73 can upregulate VEGF expression (6), thereby

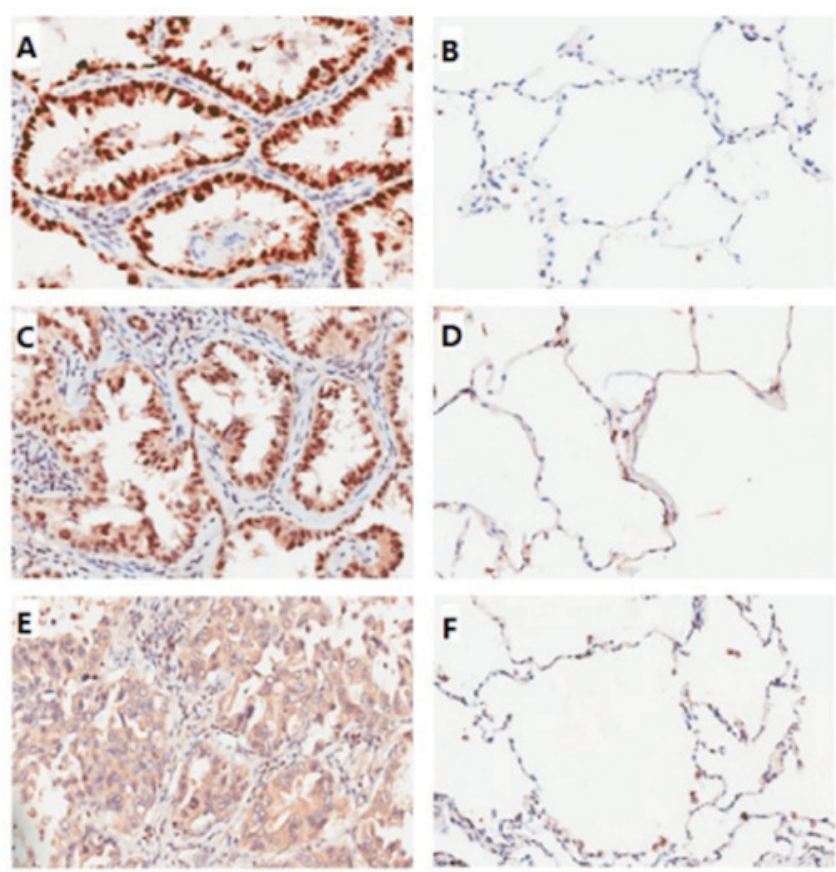

Figure 1. IHC analysis of p53, TAp73, and VASH1 expression in LAC tissues and paraneoplastic lung tissues. Micrographs show immunostaining in LAC tissues and paraneoplastic lung tissues for (A and B) p53, (C and D) TAp73, and (E and F) VASH1 expression, respectively (x200 magnification).

affecting angiogenesis; however, the specific mechanism still requires further study. Our results imply that TAp73 might function as an anti-angiogenic in LAC with mutant p53 expression. Our assumption remains to be verified in future studies.

How does TAp73 inhibit angiogenesis in LAC? It has been reported that VASH1 is a negative regulator of angiogenesis in normal tissue differentiation or tumor development. A secretory protein, VASH1 can be induced as a negative feedback regulator in endothelial cells by stimulators of angiogenesis such as VEGF and fibroblast growth factor 2 (FGF-2) (27). Many reports have indicated that VASH1 expression is high in malignant solid tumors, and that its expression is closely related to tumor angiogenesis, such as in breast (28), liver (29), renal (19), ovarian (30), colorectal (18), and lung cancer (31). Similar to these reports, we found the higher VASH1 expression in LAC tissue than in the adjacent normal 
lung tissues. More interestingly, there was a significant positive correlation between VASH1 and TAp73 expression levels similar to that between VASH1 and mutant p53. To our knowledge, there are few reports on the mechanism of TAp73 regulation of VASH1, until now. Considering TAp73 functions as a transcription factor, we used the MatInspector Database to analyze the promoter/enhancer region of VASH1, and found several potential p73 binding sites (data not shown). Whether TAp73 regulates VASH1 expression remains to be elucidated.

Collectively, our results show that TAp73, mutant p53, and VASH1 expression is significantly higher in LAC tissue compared to paraneoplastic lung tissue. Moreover, the expression trends of TAp73, mutant p53, and VASH1 are significantly positively correlated. The results imply the function of TAp73 in regulating tumor angiogenesis.

\section{Acknowledgements}

This study was supported by the National Natural Science Foundation of China (grant nos. 81071675 and 81472022), the Natural Science Foundation of Beijing (grant no. 5122021) and the Key Research Project of Medical Science of Hebei Province (grant no. 20170185). We thank Dr. Fei Pei and Dr. Min Li at the Department of Pathology, School of Basic Medical Sciences, Peking University for their help in image analysis and scoring of tissue microarray.

\section{References}

1. He YY, Zhang CX, Yang YY, Niu FY, Zeng Z, Yan HH, Xu CR, Guan JL, Zhong WZ, Yang LL, et al: Prognostic significance of genotype and number of metastatic sites in advanced non-small-cell lung cancer. Clin Lung Cancer 15: 441-447, 2014.

2. Yazdani S, Miki Y, Tamaki K, Ono K, Iwabuchi E, Abe K, Suzuki T, Sato Y, Kondo T and Sasano H: Proliferation and maturation of intratumoral blood vessels in non-small cell lung cancer. Hum Pathol 44: 1586-1596, 2013.

3. Deyoung MP and Ellisen LW: P63 and p73 in human cancer: Defining the network. Oncogene 26: 5169-5183, 2007.

4. Yang A, Walker N, Bronson R, Kaghad M, Oosterwegel M, Bonnin J, Vagner C, Bonnet H, Dikkes P, Sharpe A, et al: p73-deficient mice have neurological, pheromonal and inflammatory defects but lack spontaneous tumors. Nature 404: 99-103, 2000.

5. Marqués-Garcia F, Ferrandiz N, Fernández-Alonso R, González-Cano L, Herreros-Villanueva M, Rosa-Garrido M, Fernández-García B, Vaque JP, Marqués MM, Alonso ME, et al: p73 plays a role in erythroid differentiation through GATA1 induction. J Biol Chem 284: 21139-21156, 2009.

6. Vikhanskaya F, Bani MR, Borsotti P, Ghilardi C, Ceruti R, Ghisleni G, Marabese M, Giavazzi R, Broggini M and Taraboletti G: p73 overexpression increases VEGF and reduces thrombospondin-1 production: Implications for tumor angiogenesis. Oncogene 20: 7293-7300, 2001.

7. Guan M, Peng HX, Yu B and Lu Y: p73 Overexpression and angiogenesis in human colorectal carcinoma. Jpn J Clin Oncol 33: 215-220, 2003.

8. Petrova V, Mancini M, Agostini M, Knight RA, Annicchiarico-Petruzzelli M, Barlev NA, Melino G and Amelio I: TAp73 transcriptionally represses BNIP3 expression. Cell Cycle 14: 2484-2493, 2015.

9. Stantic M, Sakil HA, Zirath H, Fang T, Sanz G, Fernandez-Woodbridge A, Marin A, Susanto E, Mak TW, Arsenian Henriksson M and Wilhelm MT: TAp73 suppresses tumor angiogenesis through repression of proangiogenic cytokines and HIF-1 $\alpha$ activity. Proc Natl Acad Sci USA 112: 220-225, 2015.

10. Dulloo I, Phang BH, Othman R, Tan SY, Vijayaraghavan A, Goh LK, Martin-Lopez M, Marques MM, Li CW, Wang de Y, et al: Hypoxia-inducible TAp73 supports tumorigenesis by regulating the angiogenic transcriptome. Nat Cell Biol 17: 511-523, 2015.
11. Dulloo I, Hooi PB and Sabapathy K: Hypoxia-induced DNp73 stabilization regulates Vegf-A expression and tumor angiogenesis similar to TAp73. Cell Cycle 14: 3533-3539, 2015.

12. Fernandez-Alonso R, Martin-Lopez M, Gonzalez-Cano L, Garcia S, Castrillo F, Diez-Prieto I, Fernandez-Corona A, Lorenzo-Marcos ME, Li X, Claesson-Welsh L, et al: p73 is required for endothelial cell differentiation, migration and the formation of vascular networks regulating VEGF and TGF $\beta$ signaling. Cell Death Differ 22: 1287-1299, 2015.

13. Salimath B, Marmé D and Finkenzeller G: Expression of the vascular endothelial growth factor gene is inhibited by $\mathrm{p} 73$. Oncogene 19: 3470-3476, 2000.

14. Nigro JM, Baker SJ, Preisinger AC, Jessup JM, Hostetter R, Cleary K, Bigner SH, Davidson N, Baylin S, Devilee P, et al: Mutations in the p53 gene occur in diverse human tumour types. Nature 342: 705-708, 1989.

15. Zietz C, Rössle M, Haa C, Sendelhofer A, Hirschmann A, Stürzl M and Löhrs U: MDM-2 oncoprotein overexpression, p53 gene mutation, and VEGF up-regulation in angiosarcomas. Am J Pathol 153: 1425-1433, 1998.

16. Kerbel RS: Vasohibin-1: The feedback on a new inhibitor of angiogenesis. J Clin Invest 114: 884-886, 2004.

17. Kern JM, Bauer K, Rychli J, Wojta J, Ritsch A, Gastl G, Gunsilius E and Untergasser G: Alternative splicing of vasohibin-1 generates an inhibitor of endothelial cell proliferation, migration, and capillary tube formation. Arterioscler Thromb Vasc Biol 28: 478-484, 2008.

18. Yan Y, Shen Z, Ye Y, Jiang K, Zhang H, Shen C, Mustonen H, Puolakkainen P and Wang S: A novel molecular marker of prognosis in colorectal cancer: Vasohibin-1. Med Oncol 31: 816, 2014.

19. Kanomata N, Sato Y, Miyaji Y, Nagai A and Moriya T: Vasohibin-1 is a new predictor of disease-free survival in operated patients with renal cell carcinoma. J Clin Pathol 66: 613-619, 2013.

20. Lu C, Han HD, Mangala LS, Ali-Fehmi R, Newton CS, Ozbun L, Armaiz-Pena GN, Hu W, Stone RL, Munkarah A, et al: Regulation of tumor angiogenesis by EZH2. Cancer Cell 18: 185-197, 2010.

21. Li DJ, Zhou K, Wang SG, Shi Z and Yang Z: Recombinant adenovirus encoding vasohibin prevents tumor angiogenesis and inhibits tumor growth. Cancer Sci 101: 448-452, 2010.

22. Kandoth C, McLellan MD, Vandin F, Ye K, Niu B, Lu C, Xie M, Zhang Q, McMichael JF, Wyczalkowski MA, et al: Mutational landscape and significance across 12 major cancer types. Nature 502: 333-339, 2013.

23. Oren M and Rotter V: Mutant p53 gain-of-function in cancer. Cold Spring Harb Perspect Biol 2: a001107, 2010.

24. Melino G, Laurenzi VD and Vousden KH: p73: Friend or foe in tumorigenesis. Nat Rev Cancer 2: 605-615, 2002.

25. Levrero M, Laurenzi DV, Costanzo A, Gong J, Wang JY and Melino G: The p53/p63/p73 family of transcription factors: Overlapping and distinct functions. J Cell Sci 113: 1661-1670, 2000.

26. Amelio I, Inoue S, Markert EK, Levine AJ, Knight RA, Mak TW and Melino G: TAp73 opposes tumor angiogenesis by promoting hypoxia-inducible factor $1 \alpha$ degradation. Proc Natl Acad Sci USA 112: 226-231, 2015.

27. Yoshinaga K, Ito K, Moriya T, Nagase S, Takano T, Niikura H, Sasano H, Yaegashi $\mathrm{N}$ and Sato Y: Roles of intrinsic angiogenesis inhibitor, vasohibin, in cervical carcinomas. Cancer Sci 102: 446-451, 2011.

28. Tamaki K, Sasano H, Maruo Y, Takahashi Y, Miyashita M, Moriya T, Sato Y, Hirakawa H, Tamaki N, Watanabe M, et al: Vasohibin-1 as a potential predictor of aggressive behavior of ductal carcinoma in situ of the breast. Cancer Sci 101: 1051-1058, 2010.

29. Wang Q, Tian X, Zhang C and Wang Q: Upregulation of vasohibin-1 expression with angiogenesis and poor prognosis of hepatocellular carcinoma after curative surgery. Med Oncol 29: 2727-2736, 2012.

30. Takahashi Y, Koyanagi T, Suzuki Y, Saga Y, Kanomata N, Moriya T, Suzuki M and Sato Y: Vasohibin-2 expressed in human serous ovarian adenocarcinoma accelerates tumor growth by promoting angiogenesis. Mol Cancer Res 10: 1135-1146, 2012.

31. Zhang T, Yu TT, Zhang DM, Hou XM, Liu XJ, Zhao D and Shan L: Vasohibin-1 expression detected by immunohistochemistry correlates with prognosis in non-small cell lung cancer. Med Oncol 31: 963, 2014. 